\title{
Pheophorbide a-mediated photodynamic therapy induces apoptotic cell death in murine oral squamous cell carcinoma in vitro and in vivo
}

\author{
MEE-YOUNG AHN ${ }^{1}$, SEONG-MIN KWON ${ }^{1}$, YONG-CHUL KIM ${ }^{2}$, SANG-GUN AHN ${ }^{1}$ and JUNG-HOON YOON ${ }^{3}$ \\ ${ }^{1}$ Department of Pathology and Research Center for Oral Disease Regulation of the Aged, School of Dentistry, \\ Chosun University, Gwangju 501-759; ${ }^{2}$ Department of Life Sciences, Gwangju Institute of Science and \\ Technology, Gwangju 500-712; ${ }^{3}$ Department of Oral and Maxillofacial Pathology, College of Dentistry, \\ Daejeon Dental Hospital, Wonkwang University, Daejeon 302-120, Republic of Korea
}

Received December 27, 2011; Accepted February 15, 2012

DOI: $10.3892 / o r .2012 .1748$

\begin{abstract}
Photodynamic therapy (PDT) with several photosensitizers is a promising modality for the treatment of cancer. In this study, the therapeutic effect of PDT using the synthetic photosensitizer pheophorbide a (Pa-PDT) was examined in AT-84 murine oral squamous cell carcinoma (OSCC) cells. The MTT assay revealed that Pa-PDT induced cell growth inhibition in a dose- and time-dependent manner. Pa-PDT treatment significantly induced intracellular ROS generation, which is critical for cell death induced by Pa-PDT. Cell cycle analysis showed the increased sub-G1 proportion of cells in Pa-PDT-treated cells. Induction of apoptotic cell death was confirmed by DAPI staining and the reduction of mitochondrial membrane potential $(\Delta \Psi \mathrm{m})$ on Pa-PDT-treated cells. The changes in apoptosis-related molecules were next examined using western blotting. Cytochrome c release and cleavage of caspase- 3 and PAPR were observed in AT- 84 cells, whereas Bcl-2 protein levels were decreased. To determine the therapeutic effect of Pa-PDT in vivo, a murine OSCC animal model was used. Treatment of mice with Pa-PDT significantly inhibited tumor growth, especially PDT with $\mathrm{Pa}$ intravenous administration (i.v. Pa-PDT), and increased proliferative cell nuclear antigen (PCNA) levels and TUNEL-stained apoptotic cells compared to vehicle-treated controls. The data demonstrate that the in vitro effects of Pa-PDT on the inhibition of
\end{abstract}

Correspondence to: Dr Sang-Gun Ahn, Department of Pathology, School of Dentistry, Chosun University, 375 Dong-gu, Seoseokdong, Gwangju 501-759, Republic of Korea

E-mail: ahnsg@chosun.ac.kr

Dr Jung-Hoon Yoon, Department of Oral and Maxillofacial Pathology, College of Dentistry, Daejeon Dental Hospital, Wonkwang University, Daejeon 302-120, Republic of Korea

E-mail: opathyoon@wku.ac.kr

Key words: pheophorbide a, photodynamic therapy, oral squamous cell carcinoma tumor cell proliferation and induction of apoptosis correlate to the anticancer activity of Pa-PDT in vivo. Our findings suggest the therapeutic potential of Pa-PDT in OSCC.

\section{Introduction}

Oral squamous cell carcinoma (OSCC) is the most common cancer of the the oral and maxillofacial region and accounts for over 300,000 new cancer cases worldwide annually. Despite the great advance of modern surgical techniques combined with various adjuvant treatment modalities, such as radiotherapy and chemotherapy, patients are often diagnosed at late stages and the 5-year survival rate has remained relatively low at only approximately 50\% (1-3). However, many severe side effects appear in patients receiving these treatments (4). Therefore, developing alternative therapies for oral cancer is required.

Photodynamic therapy (PDT) is used as a therapeutic alternative for treating a variety of malignant tumors (5). PDT typically involves the systemic administration of a photosensitizer followed by its activation by light of an appropriate wavelength to create a photochemical reaction $(6,7)$. Most types of cancers are especially active in both the uptake and accumulation of photosensitizer agents than normal tissues, which makes tumors especially vulnerable to PDT (8). PDT employs a photosensitizer and visible light to produce singlet oxygen and other reactive oxygen species, which cause an oxidative stress in cells and membrane damage which eventually leads to cell death and tumor ablation (9-11). PDT was reported to inhibit cancer through different cell death pathways and its effectiveness is dependent on the types of cancer cell lines and photosensitizers (12-14). During light exposure, photosensitizers that are localized in mitochondria may induce apoptosis, while those localized in lysosomes and cell membranes may cause necrosis.

The type of photosensitizer is one of the important components to determine optical photodynamic efficacy. Although several photosensitizers have been developed, limited photosensitizers such as porphyrin derivatives have been used for clinical PDT and still need to be improved in terms of their selective localization and absorption for tumors. A growing 
number of second generation photosensitizers are also undergoing clinical evaluation (14).

Pheophorbide a $(\mathrm{Pa})$ is a chlorine based photosensitizer derived from chlorophyll-a and is one of the active components of an ethnopharmacological herb Scutellaria barbata which is commonly used for tumor treatments in Asia (15). Our group recently synthesized $\mathrm{Pa}$ by the removal of a magnesium ion and a phytyl group from chlorophyll-a (16). The maximum excitation wavelength for $\mathrm{Pa}$ is around 675-683 nm, so $\mathrm{Pa}$ exhibits high photon absorption at a longer wavelength and can penetrate tissues more deeply compared to other photosensitizers, such as photofrin $(17,18)$. The energy production with the use of $\mathrm{Pa}$ is smaller than that with other photosensitizers, which have to be excited at shorter wavelengths, therefore causing less harm to the surrounding normal tissues (19). Recent studies reported that Pa-based PDT can effectively inhibit tumor cell proliferation in several cancer cells including hepatocellular carcinoma, colon cancer, pigmented melanoma and breast adenocarcinoma $(15,18,19)$. However, Pa-PDT treatment in OSCC has been rarely studied. In this study, the antitumor efficacy of PDT with newly synthesized $\mathrm{Pa}$ was evaluated in murine OSCC cells through both in vitro and in vivo experiments.

\section{Materials and methods}

Preparation of pheophorbide $a$. Pa was synthesized according to the procedure previously described (16). Briefly, treatment of the ethanol solution of chlorophyll $\mathrm{a}$ in an acidic condition (1 $\mathrm{N} \mathrm{HCl}, \mathrm{pH} 2.5$ ) enabled to remove the $\mathrm{Mg}^{2+}$ ion easily to afford a crude pheophytin in the form of precipitates. The pheophytin was subsequently hydrolyzed by reacting with $80 \%$ TFA in water to obtain $\mathrm{Pa}$ as a fine powder.

Cell culture. AT-84 cells, murine OSCC cell lines, were maintained in RPMI medium (Invitrogen, Eugene, OR, USA) containing $10 \%$ fetal bovine serum (Invitrogen), $100 \mathrm{U} / \mathrm{ml}$ penicillin-streptomycin (Welgene, Daegu, South Korea) and subsequently incubated at $37^{\circ} \mathrm{C}$ in an atmosphere containing $5 \% \mathrm{CO}_{2}$. AT-84 cells were kindly provided by Dr E.J. Shillitoe (State University of New York, Upstate Medical University) and have been previously described (20).

Photodynamic treatment. AT-84 cells were incubated in serum-free culture medium with $\mathrm{Pa}$ for $2 \mathrm{~h}$. After washing, the cells were exposed to a light dose of $4.24 \mathrm{~J} / \mathrm{cm}^{2}$ performed using a laser diode (Geumgwang Co., Ltd., Daejeon, Korea) at a wavelength of $664 \mathrm{~nm}$.

MTT assay. The viability of AT- 84 cells was assessed by the MTT assay. Cells $\left(2 \times 10^{4}\right.$ cells/well) were seeded in 24-well plates and incubated for $24 \mathrm{~h}$ to allow attachment. After washing with PBS, the cells were incubated with Pa for $2 \mathrm{~h}$, followed by irradiation. After 24 and $48 \mathrm{~h}$, the cells were washed twice with PBS, and $0.5 \mathrm{ml}$ of cell culture medium and $50 \mu \mathrm{l}$ of 3-(4,5-dimethylthiazol-2-yl)-3-5-diphenyltetrazolium bromide solution $5 \mathrm{mg} / \mathrm{ml}$ (Amresco, Solon, OH, USA) were added. After $4 \mathrm{~h}$ of incubation, the medium was removed and $250 \mu \mathrm{l}$ of dimethyl sulfoxide (DMSO) was added. The absorbance was measured at $595 \mathrm{~nm}$ with a Microplate Autoreader
ELISA (Beckamn Coulter, Wals, Austria) and all experiments were performed in triplicate.

Measurement of intracellular reactive oxygen species (ROS). After Pa-PDT, the cells were washed twice with PBS and incubated in medium containing $10 \mu \mathrm{M}$ of $2^{\prime} 7^{\prime}$-dichlorofluorescein diacetate $\left(\mathrm{H}_{2} \mathrm{DCFDA}\right.$; Invitrogen) at $37^{\circ} \mathrm{C}$ in an atmosphere containing $5 \% \mathrm{CO}_{2}$ for $30 \mathrm{~min}$. Fluorescence was analyzed immediately by Thermo Scientific Varioskan ${ }^{\circledR}$ Flash spectral scanning multimode reader (Varioskan, Thermo Electron Co.) with SkanIt ${ }^{\circledR}$ Software (wavelength: excitation 450-495, emission 517-527).

Measurement of intracellular singlet oxygen. The cells were gently rinsed with PBS at 3 min after Pa-PDT and incubated with $10 \mu \mathrm{M}$ trans-1-(2'-methoxyvinyl) pyrene (Invitrogen) in the dark for $15 \mathrm{~min}$ at $37^{\circ} \mathrm{C}$. Fluorescence was analyzed immediately by Thermo Scientific Varioskan ${ }^{\circledR}$ Flash spectral scanning multimode reader (Varioskan, Thermo Electron Co.) with SkanIt ${ }^{\circledR}$ Software (wavelength: excitation 450-495, emission 517-527).

Detection of change in mitochondrial membrane potential $\left(\Delta \Psi_{m}\right)$. The cells $\left(3 \times 10^{5}\right)$ were seeded on each well of a 6 -well culture plate and incubated $24 \mathrm{~h}$ to allow attachment. After Pa-PDT treatment, the media was removed and the cells were incubated with fresh culture medium containing $2.5 \mu \mathrm{g} / \mathrm{ml}$ JC-1 dye (Invitrogen) for $30 \mathrm{~min}$ at $37^{\circ} \mathrm{C}$ in the dark. Cells on coverslips were washed with PBS twice and fixed with $2.5 \%$ paraformaldehyde made in $200 \mathrm{mM}$ HEPES buffer for $15 \mathrm{~min}$ at room temperature followed by PBS wash. The cells were then analyzed for JC-1 uptake by using an Olympus IX71 (Tokyo, Japan) fluorescence microscope.

Flow cytometry. At $24 \mathrm{~h}$ after Pa-PDT, $1 \times 10^{6}$ AT-84 cells were stained with the Apoptosis Assay kit (Invitrogen), followed by labeling with Alexa Fluor ${ }^{\circledR} 488$ Annexin V and propidium iodide. The 10,000 cells per sample were analyzed immediately using the Cell Lab Quanta ${ }^{\mathrm{TM}}$ SC flow cytometer (Beckman Coulter) and software.

Western blotting. The total protein was extracted using lysis buffer (1\% Triton X-100, $150 \mathrm{mM} \mathrm{NaCl}, 5 \mathrm{mM}$ EDTA, and protease inhibitors). Cytosol protein was extracted and equal amounts of protein were electrophoresed on an SDS-PAGE gel and transferred to a PVDF membrane (Millipore, Billerica, MA, USA). After blocking in TBS-T (20 mmol/1 Tris, $137 \mathrm{mmol} / 1 \mathrm{NaCl}, 1 \mathrm{~g} / 1$ Tween-20, pH 7.6) with 5\% skim milk for $1 \mathrm{~h}$ at room temperature, the membranes were incubated with primary antibodies against cleaved caspase-3, cleaved caspase-9, PARP (Cell Signaling, Beverly, MA, USA), Bcl-2, cytochrome $c$ and actin (Santa Cruz Biotechnology, Santa Cruz, CA, USA) overnight at $4^{\circ} \mathrm{C}$. The membranes were then washed three times with TBS-T and incubated with secondary antibodies (Santa Cruz Biotechnology) for $1 \mathrm{~h}$ at room temperature. Finally, the membranes were visualized using the ECL detection reagent (Millipore).

In vivo study of Pa-PDT on AT-84 cells-bearing C3H mice. AT-84 cells were used for an in vivo experiment, which was 

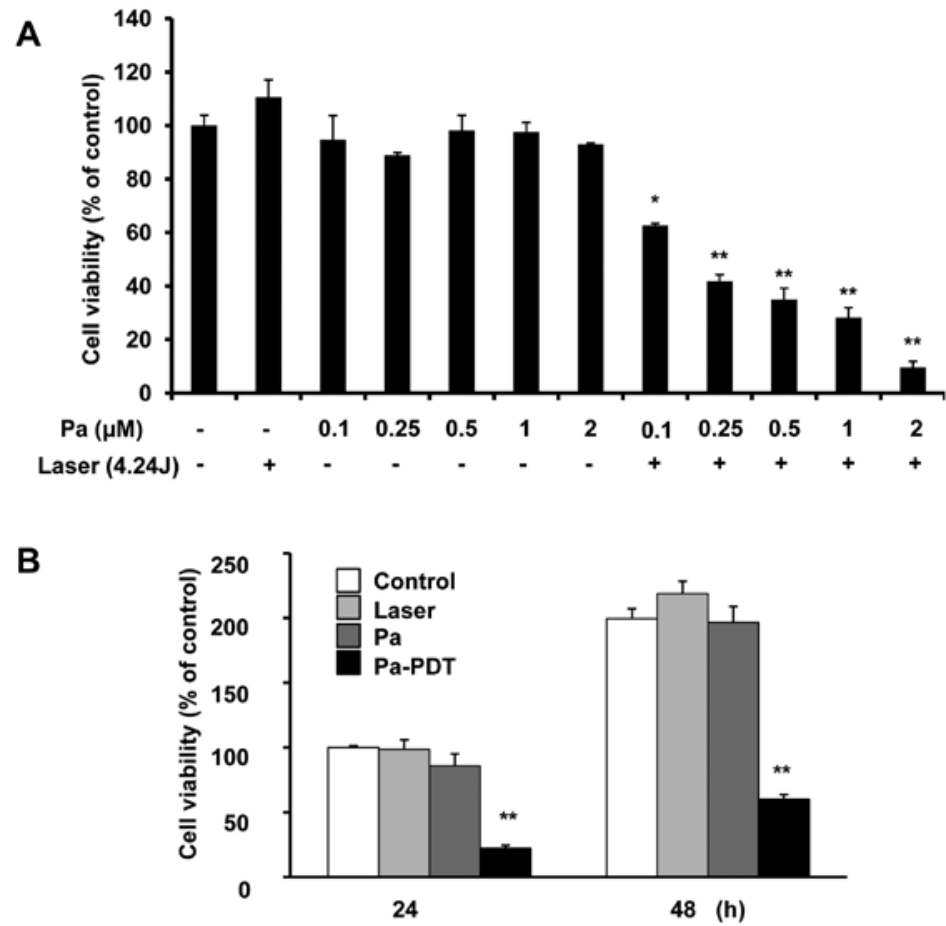

Figure 1. Pa-PDT induces growth inhibition of AT-84 cells. (A) AT-84 cells were incubated with the indicated concentrations of Pa followed by exposure to $4.24 \mathrm{~J} / \mathrm{cm}^{2}$ of laser light for $24 \mathrm{~h}$. (B) AT-84 cells were incubated with $0.25 \mu \mathrm{M}$ of Pa followed by exposure to $4.24 \mathrm{~J} / \mathrm{cm}^{2}$ of laser light for the indicated time periods. The data is reported as the mean $\pm \mathrm{SD}$ of three independent experiments. ${ }^{*} \mathrm{P}<0.05,{ }^{* *} \mathrm{P}<0.01$ compared to untreated control.

previously established by Pang et al (21). Six-week-old male immunocompetent C3H mouse (Samtaco, Sungnam, Korea) were inoculated subcutaneously on the right flank with $1 \times 10^{6}$ AT-84 cells. One week later, the animals were treated with a $10 \mathrm{mg} / \mathrm{kg}$ intravenous (i.v.) or a $30 \mathrm{mg} / \mathrm{kg}$ intraperitoneal (i.p.) injection of $\mathrm{Pa}$. After $24 \mathrm{~h}$, PDT was performed using a laser diode at a light dose of $100 \mathrm{~J} / \mathrm{cm}^{2}$ and wavelength of $664 \mathrm{~nm}$. The animals were monitored daily and the tumor volume was measured by caliper and calculated by the formula: $\mathrm{V}=$ $\left(a b^{2}\right) / 2$, in which $a$ is the longest diameter and $b$ is the shortest diameter of the tumor. All experiments were performed under protocols approved by the Animal Care and Use Committee at the Chosun University, School of Dentistry.

Histopathology, immunohistochemistry and TUNEL assay. The animals were killed on Day 15 and the tumors were removed carefully and fixed in $10 \%$ formalin over $24 \mathrm{~h}$. The tissues were then dehydrated in an alcohol-xylene series and embedded in paraffin wax. From each block, $2 \mu \mathrm{m}$ sections were prepared and stained with hematoxylin (Vector, Burlingame, CA, USA) and eosin for histological examination. For immunohistochemistry, the sections were incubated in $3 \% \mathrm{H}_{2} \mathrm{O}_{2}$ in methanol for $10 \mathrm{~min}$ to remove endogenous peroxidase and blocked with $1 \%$ BSA in PBS for $1 \mathrm{~h}$. The sections were then incubated with anti-PCNA antibody (Dako, Carpinteria, CA, USA) overnight at $4^{\circ} \mathrm{C}$. After three washes with PBS-T, the sections were subjected to the avidin-biotin peroxidase complex (ABC) method (Vector) and peroxidase activity was evaluated with 3,3'-diaminobenzidine (Vector). Finally, the sections were counterstained with hematoxylin. The terminal deoxynucleotidyl transferase-mediated dUTP nick end labeling (TUNEL) assay was performed using an
Apoptosis Detection kit (Millipore) according to the manufacturer's protocol. PCNA-positive cells and apoptotic cells were counted from five randomly selected areas under x200 magnifications and represented as mean $\pm \mathrm{SD}$.

Statistical analysis. All statistical analyses were carried out using the Excel software. The significance for the difference was determined by independent-samples t-test. A P-value $<0.05$ was regarded statistically significant.

\section{Results}

Inhibition of cellular proliferation by Pa-PDT in AT-84 cells. The cellular uptake of Pa in AT-84 cells was observed after $30 \mathrm{~min}$ and up to $24 \mathrm{~h}$ of treatment with $0.25 \mu \mathrm{M} \mathrm{Pa}$ (data not shown). Pa administration for 2-h was selected for subsequent experiments. To examine the effects of Pa-PDT on cell proliferation, the cells were treated with various doses of $\mathrm{Pa}$ for $24 \mathrm{~h}$. Pa-PDT significantly inhibited proliferation in dose-dependent manner up to $2 \mu \mathrm{M} \mathrm{Pa}(\mathrm{P}<0.001)$. However, there was no cytotoxicity in cells treated with $\mathrm{Pa}$ or laser alone (Fig. 1A). The $\mathrm{IC}_{50}$ value for Pa-PDT was $0.25 \mu \mathrm{M}$. The cell viability was also time-dependently decreased in $0.25 \mu \mathrm{m}$ Pa-PDT-treated AT-84 cells (Fig. 1B).

ROS production and mitochondrial membrane potential change by $P a-P D T$. PDT can trigger the generation of ROS by exciting the photosensitizer, and the produced high-energy molecules can collapse the subcellular organelles. As shown in Fig. 2A, ROS production was significantly induced after Pa-PDT in AT-84 cells. We also found that the intracellular singlet oxygen level was increased immediately after Pa-PDT 
A

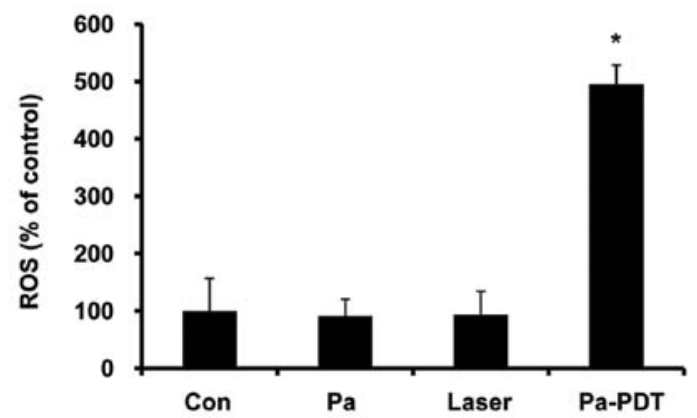

B

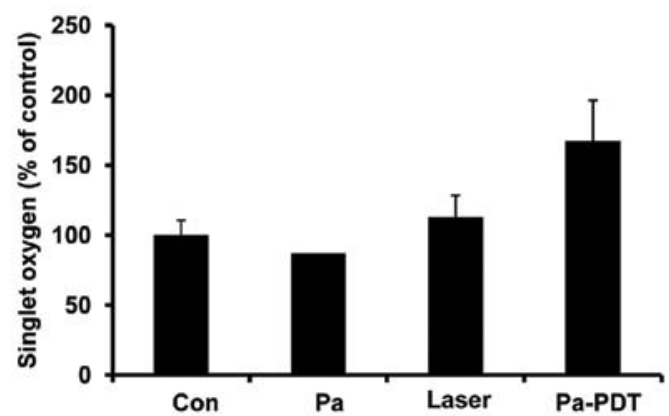

C
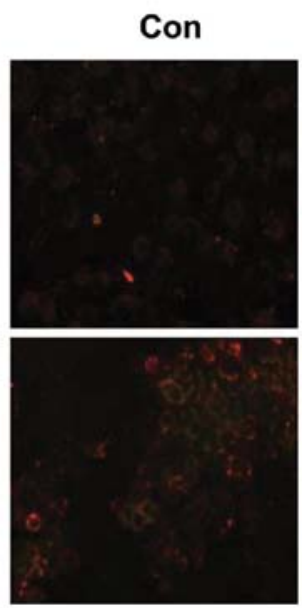

Laser
$\mathrm{Pa}$
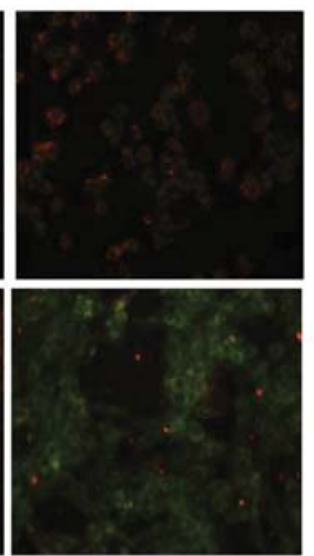

Pa-PDT

Figure 2. Pa-PDT increases ROS and singlet oxygen and reduces mitochondrial membrane potential on AT- 84 cells. AT- 84 cells were incubated with $0.25 \mu \mathrm{M}$ of Pa followed by exposure to $4.24 \mathrm{~J} / \mathrm{cm}^{2}$ of laser light. Quantitative amount of ROS production (A) and singlet oxygen (B) was measured using the scanning multimode reader and software. (C) AT-84 cells were seeded, incubated for $24 \mathrm{~h}$, and Pa-PDT for $24 \mathrm{~h}$. Then, cells were stained with the JC-1 mitochondrial membrane potential indicator specific dye, and analyzed using fluorescence microscopy. The data reported are the mean \pm SD of three independent experiments. "P $<0.05$ compared to untreated control.

A

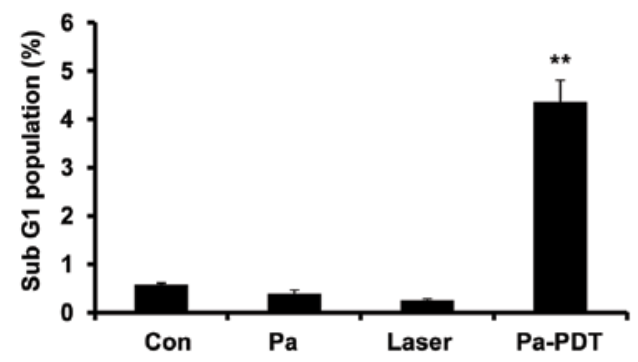

B

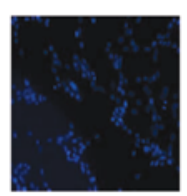

Con

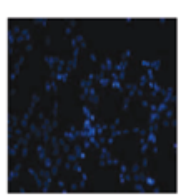

$\mathrm{Pa}$

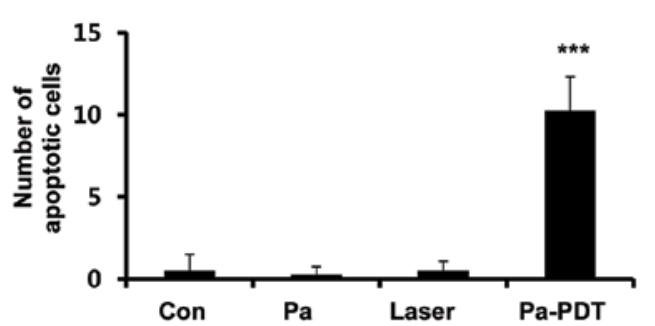

Laser Pa-PDT
C

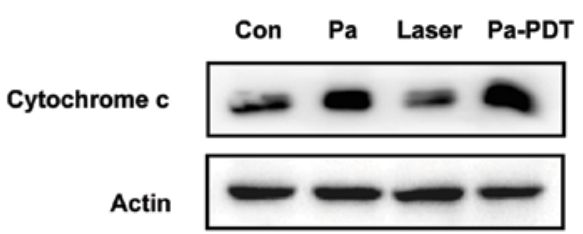

D
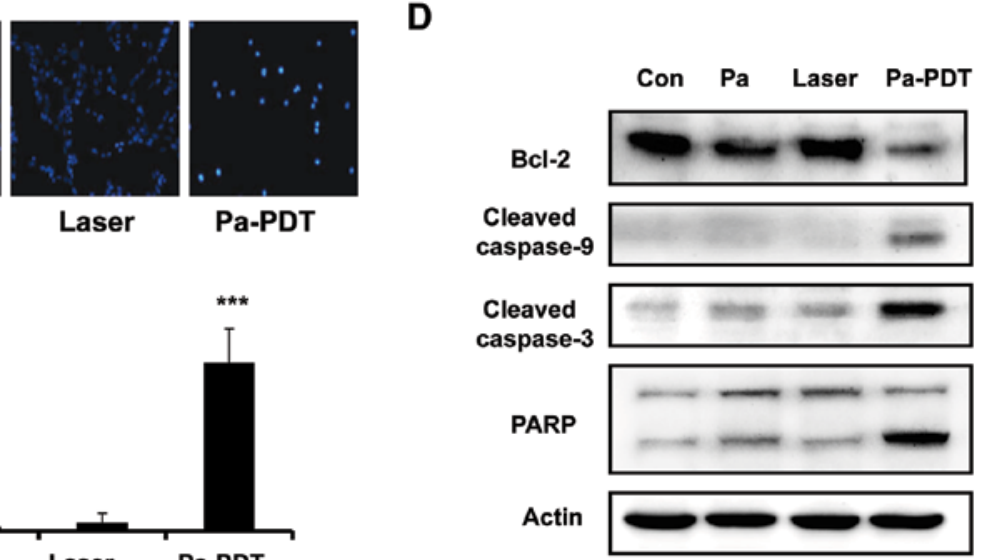

Figure 3. Pa-PDT induces the apoptosis through a caspase-dependent pathway in AT- 84 cells. AT- 84 cells were incubated with $0.25 \mu \mathrm{M}$ of Pa for $2 \mathrm{~h}$ followed by exposure to $4.24 \mathrm{~J} / \mathrm{cm}^{2}$ of laser light. (A) Apoptosis was evaluated by the flow cytometric method by staining the cells with PI. (B) Apoptotic bodies were observed in Pa-PDT treated AT-84 cells for $24 \mathrm{~h}$ by DAPI staining. Morphological patterns of apoptosis in AT-84 cells exposed to Pa-PDT. The data are expressed as the means $\pm \mathrm{SD}$ of three independent experiments. ${ }^{* *} \mathrm{P}<0.01,{ }^{* * *} \mathrm{P}<0.001$ compared to untreated control. (C and $\left.\mathrm{D}\right)$ The protein fraction was prepared and resolved by 10-12\% SDS-PAGE. The expression levels of cytochrome c (cytosol fraction), Bcl-2, cleaved caspase-3, cleaved caspase-9, cleaved PARP, and actin were detected by western blot analysis. The representative bands from three independent experiments are shown.

treatment, compared with the control, Pa or laser alone group (Fig. 2B). The mitochondrial membrane potential change was next measured by JC-1 staining, which exhibits potentialdependent accumulation in mitochondria. The fluorescence 
A
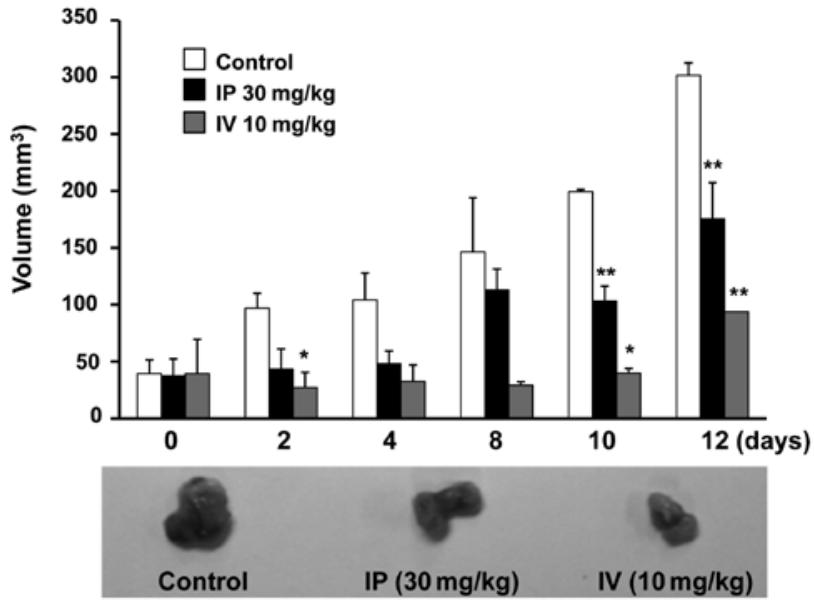

B

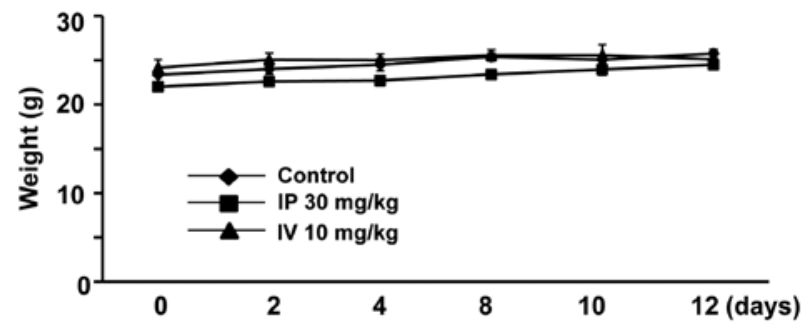

Figure 4. Pa-PDT inhibits tumor growth in vivo. AT- 84 cells $\left(5 \times 10^{6} \mathrm{per}\right.$ mouse) were injected subcutaneously into the right flank of $\mathrm{C} 3 \mathrm{H}$ mice. (A and B) Tumor volume and body weight was measured every 2 days and calculated using the formula $\mathrm{V}=\left(\mathrm{ab}^{2}\right) / 2$, in which $\mathrm{a}$ is the largest diameter and $\mathrm{b}$ is the shortest diameter of the tumor. Results are expressed as mean $\pm \mathrm{SD} .{ }^{*} \mathrm{P}<0.05$, ${ }^{* *} \mathrm{P}<0.01$ compared to untreated control.

emission of Pa-PDT-treated cells shifted from red $(\sim 590 \mathrm{~nm})$ to green ( 529 nm) (Fig. 2C). Consequently, mitochondrial depolarization was indicated by an increase in the green/red fluorescence intensity ratio.
Apoptotic cell death by Pa-PDT. We next investigated the effects of Pa-PDT on cell death pathway using cell cycle analysis and DAPI staining. The population of sub-G1 phase cells was increased in Pa-PDT-treated AT-84 cells (Fig. 3A). As shown in Fig. 3B, enhanced DAPI fluorescence by condensed or fragmented chromatin was observed in cells treated with Pa-PDT, as compared to the control, Pa or laser alone group. The expression levels of apoptosis related proteins were next measured to determine the apoptotic mechanism of Pa-PDT by western blotting. The levels of cytochrome $\mathrm{c}$ were increased in a cytosol fraction of Pa-PDT-treated cells (Fig. 3C). In addition, Pa-PDT dramatically reduced the expression of $\mathrm{Bcl}-2$, anti-apoptotic gene, and upregulated the activated form of caspase-3. Pa-PDT also increased the levels of PARP cleavage, a known endogenous substrate for caspases that plays important roles in apoptosis in AT-84 cells (Fig. 3D).

In vivo study of Pa-PDT on AT-84 cells. The effect of Pa-PDT on in vivo turmor growth was monitored using the $\mathrm{C} 3 \mathrm{H}$ mouse model with subcutaneous injection of AT- 84 cells. Pa was administered i.v. or i.p. to AT-84 cells-inoculated mice. After $24 \mathrm{~h}$, PDT was performed using a laser diode and tumor volume was measured every other day after Pa-PDT treatment. As shown in Fig. 4A, Pa-PDT, especially i.v. Pa-PDT, significantly inhibited tumor growth up to $70 \%$, while the i.p. Pa-PDT-induced inhibition was up to $43.4 \%$ relative to the control group at the end of period. No systemic toxicity including the body weight changes or other apparent adverse effects were observed in the animals throughout the study period (Fig. 4B).

Immunohistochemical expression of PCNA and TUNEL assay. The histological changes between the Pa-PDT treated and control group were examined using $\mathrm{H} \& \mathrm{E}$ staining. As shown in Fig. 5A, the tumor tissues treated with Pa-PDT had
A

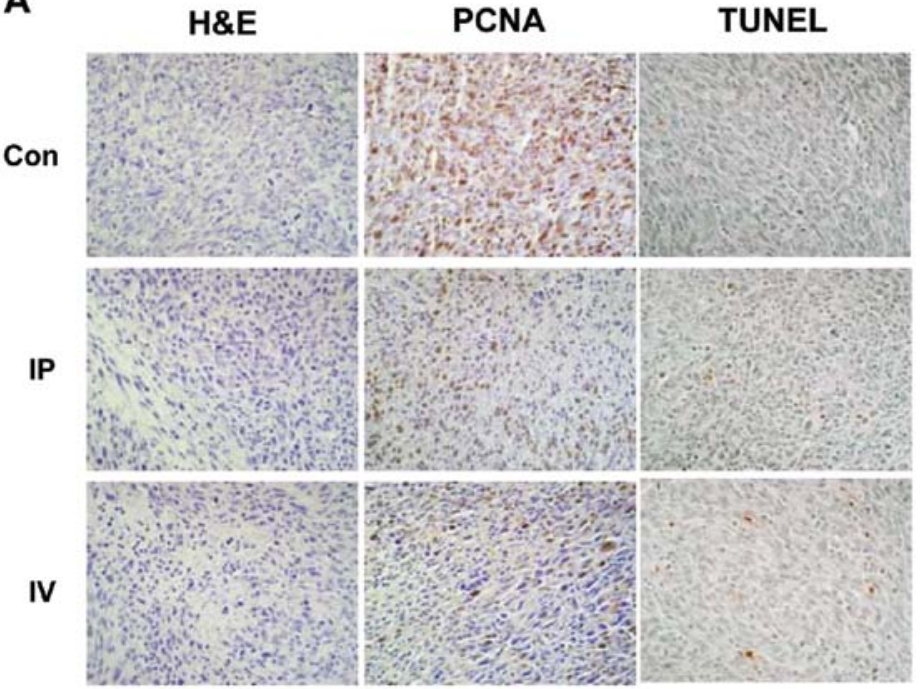

B
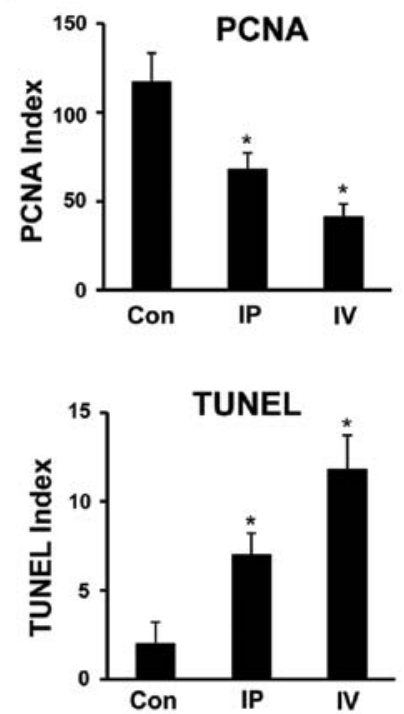

Figure 5. Pa-PDT decreases the proliferation and induces apoptosis of the tumor in vivo. (A) H\&E staining of tumor sections (left), immunohistochemistry for PCNA (middle), and TUNEL assay (right) were performed on paraffin sections from the tumor. (B) Positive cells for PCNA immunostaining (top) and TUNEL (bottom) were counted and results are expressed. The data are reported as the means \pm SD of three independent experiments. ${ }^{*}<0.05$ compared to the untreated control. 
larger areas of extensive cell death showing nuclear pyknosis and cytoplasmic eosinophilia, whereas the control tumor tissues demonstrated solid growth of SCC with hemorrhage.

The antitumor effects of Pa-PDT in the in vivo study were next investigated by examining the expression of PCNA, a cell proliferation marker, and TUNEL staining for assessing the amount of apoptotic cells, by immunohistochemistry. The number of PCNA-positive cells significantly decreased in tumor treated with both i.v. and i.p. Pa-PDT compared to control. TUNEL-positive cells were dramatically increased in Pa-PDT treated tumor tissues compared to untreated control. The tumor tissues treated with i.v. Pa-PDT demonstrated higher expression of PCNA and fewer TUNEL-positive cells than i.p. Pa-PDT treated tumor tissues (Fig. 5B). These results showed that the in vitro effect of Pa-PDT on the inhibition of tumor cell proliferation and apoptotic cell death correlates with the antitumor activity of Pa-PDT in vivo.

\section{Discussion}

PDT is a revolutionary treatment aimed at detecting cancers and treating them without surgery or chemotherapy. It is based on the discovery that certain chemicals known as photosensitizing agents can induce cell death when the cells are exposed to a particular type of light. In this study, the therapeutic effect of PDT using the synthetic photosensitizer Pa was examined in murine OSCC AT-84 cells. We used newly synthesized Pa which may be more easily available for commercial usage compared to purified $\mathrm{Pa}$ from plants.

The cytotoxic effect of PDT with $\mathrm{Pa}$ was first performed in AT-84 cells. No cytotoxic effect was observed in Pa or laser alone treated cells, but Pa-PDT significantly inhibited cell proliferation in dose-dependent manner. Because the cytotoxic effects of PDT appear to arise from the generation of singlet oxygen and free radicals due to a photodynamic reaction, tumor oxygenation should be an important factor for the antitumor effects of PDT $(9,22,23)$. The type I radicals and type II ROS such as singlet oxygen have been implicated in the therapeutic or toxic responses of PDT (24). We next examined the levels of ROS including singlet oxygen in Pa-PDT treated cells. The levels of ROS and singlet oxygen were immediately increased after Pa-PDT treatment. Singlet oxygen released from Pa-PDT mediated mitochondrial damage (15). We also determined the mitochondrial membrane potential variations $(\Delta \Psi \mathrm{m})$ with use of the fluorescence probe JC-1, the most widely implemented application for detecting mitochondrial depolarization (25). The result showed that the mitochondrial membrane potential was rapidly altered in Pa-PDT-treated cells thus indicating that the mitochondrial membrane was damaged, resulting in alterations to membrane permeability.

Highly toxic singlet oxygen and oxygen radicals can react with cellular components to cause cell death (26). Although PDT induced cell death in a variety of cancers, the molecular mechanisms involved in each death event may differ $(24,27)$. Rapozzi et al showed that a high dose of Pa-PDT caused apoptotic morphological changes in HeLa cells, but necrotic morphological changes in HepG2 cells (28). We next investigated the cell death mechanism by Pa-PDT. It became evident that mitochondria are also active participants in apoptosis. Collapse or depolarization of mitochondria is often, but not always, one of the first measurable events during apoptosis and the release of cytochrome $\mathrm{c}$ into the cytosol activates the intrinsic apoptotic pathway to trigger cell death (29). In our study, Pa-PDT treatment significantly increased apoptotic cells as determined by FACS (sub-G1 population) and DAPI staining. The amount of cytochrome $\mathrm{c}$ in the cytosolic fraction was also increased and the level of anti-apoptotic Bcl-2 was decreased after Pa-PDT treatment in AT-84 cells, as expected. Although the cytosolic cytochrome c was slightly increased in $\mathrm{Pa}$ alone-treated cells, this was not accompanied by apoptotic cell death assessed by DAPI staining or by changes in the sub-G1 proportion with $\mathrm{Pa}$ alone treatment. Previous studies have shown that the fluorescence of $\mathrm{Pa}$ was distributed only in the cytoplasm, but not in nuclei indicating that $\mathrm{Pa}$ largely acts on the intrinsic apoptotic pathway $(19,30)$. Consistent with these observations, our results demonstrated that Pa-PDT significantly led to activation of caspase-9, caspase-3 and PARP cleavage in AT-84 cells. All these data imply that induction of intracellular ROS was accompanied by depolarization of the mitochondrial membrane potential followed by the release of cytochrome $\mathrm{c}$ from the mitochondria to the cytosol, caspase activation and other events that lead to apoptosis (29).

We next examined the effect of Pa-PDT on in vivo tumor growth using this mouse model with subcutaneous injection of AT-84 cells. The route of photosensitizer administration affected the efficacy of PDT. The i.v. route of photosensitizer administrations are widely used in clinical and preclinical applications of PDT (31). Intraperitoneal PDT is currently being evaluated in patients for the treatment of carcinomatosis and sarcomatosis (32). In this study, $\mathrm{Pa}$ was given once i.v. or i.p. in a mouse tumor model and PDT light was administered using a laser diode after $24 \mathrm{~h}$. As a result, Pa-PDT significantly decreased the tumor volume in both i.v. and i.p. administrations of $\mathrm{Pa}$ compared to control group. These results suggest that only a single application of Pa-PDT can effectively inhibit the progression of a solid tumor in vivo. Despite the fact that the $\mathrm{Pa}$ dosage for i.p. injection was three times higher than the i.v. injection considering $\mathrm{Pa}$ tissue distribution differences (33), i.v. administration of $\mathrm{Pa}$ more effectively inhibited tumor growth than i.p. administration of $\mathrm{Pa}$. This result supports that i.v. Pa-PDT is the optimal administration route for $\mathrm{Pa}$ in in vivo assay systems.

Based on an in vitro study, we focused on examining the inhibition of cell proliferation and apoptotic cell death in Pa-PDT treated tumor tissues. Treatment of Pa-PDT decreased AT-84 tumor cell proliferation, as assessed by immunohistological detection of the proliferation marker, PCNA. We also performed TUNEL staining to assess the amount of the apoptotic cells in vivo. TUNEL-positive cells significantly increased in tumors treated with Pa-PDT, especially i.v. Pa-PDT, compared with the control group. These results confirmed data that Pa-PDT effectively arrests the tumor growth by inhibiting cell proliferation and inducing apoptosis. In addition, i.v. Pa-PDT treatment led to a decrease of the proliferating index and an increase of the apoptotic index compared to i.p. Pa-PDT, as evident from the tumor volume change.

In conclusion, these results show that the PDT using newly synthesized photosensitizer $\mathrm{Pa}$ induced cell growth inhibition 
and intracellular ROS generation. Pa-PDT promoted apoptotic cell death by regulating mitochondrial integrity and caspase activity. These potent antitumor activities of Pa-PDT were observed in both the in vitro and the in vivo experiments. Therefore, these findings suggest that Pa-PDT could be a potent clinical therapeutic strategy for OSCC.

\section{Acknowledgements}

This study was supported by a grant of the Korean Health Technology R\&D Project, Ministry for Health, Welfare and Family Affairs, Republic of Korea (no. A100490).

\section{References}

1. Lippman SM, Sudbo J and Hong WK: Oral cancer prevention and the evolution of molecular-targeted drug development. J Clin Oncol 23: 346-356, 2005.

2. Rautava J, Jee KJ, Miettinen PJ, et al: ERBB receptor in developing, dysplastic and malignant oral epithelia. Oral Oncol 44: 227-235, 2008 .

3. Scully C and Bagan J: Oral squamous cell carcinoma overview. Oral Oncol 45: 301-308, 2009.

4. Hopper C, Kübler A, Lewis H, Tan IB and Putnam G: mTHPCmediated photodynamic therapy for early oral squamous cell carcinoma. Int J Cancer 111: 138-146, 2004.

5. Dougherty TJ, Gomer CJ, Henderson BW, et al: Photodynamic therapy. J Natl Cancer Inst 90: 889-905, 1998.

6. Grimm S, Mvondo D, Grune T and Breusing N: The outcome of 5-ALA-mediated photodynamic treatment in melanoma cells is influenced by vitamin $\mathrm{C}$ and heme oxygenase-1. Biofactors 37 : 17-24, 2011.

7. Moon YH, Park JH, Kim SA, Lee JB, Ahn SG and Yoon JH: Anticancer effect of photodynamic therapy with hexenyl ester of 5-aminolevulinic acid in oral squamous cell carcinoma. Head Neck 32: 1136-1142, 2010.

8. Selbo PK, Hogset A, Prasmickaite L and Berg K: Photochemical internalisation: a novel drug delivery system. Tumour Biol 23: 103-112, 2002.

9. Weishaupt KR, Gomer CJ and Dougherty TJ: Identification of singlet oxygen as the cytotoxic agent in photoinactivation of a murine tumor. Cancer Res 36: 2326-2329, 1976.

10. Moan J and Berg K: Photochemotherapy of cancer: experimental research. Photochem Photobiol 55: 931-948, 1992.

11. Chiu S and Oleinick NL: Dissociation of mitochondrial depolarization from cytochrome c release during apoptosis induced by photodynamic therapy. Br J Cancer 84: 1099-1106, 2001.

12. Buytaert E, Dewaele M and Agostinis P: Molecular effectors of multiple cell death pathways initiated by photodynamic therapy. Biochim Biophys Acta 1776: 86-107, 2007.

13. Zuluaga MF and Lange N: Combination of photodynamic therapy with anti-cancer agents. Curr Med Chem 15: 1655-1673, 2008.

14. Luna MC, Ferrario A, Wong S, Fisher AM and Gomer CJ: Photodynamic therapy-mediated oxidative stress as a molecular switch for the temporal expression of genes ligated to the human heat shock promoter. Cancer Res 60: 1637-1644, 2000.

15. Tang PM, Liu XZ, Zhang DM, Fong WP and Fung KP: Pheophorbide a based photodynamic therapy induces apoptosis via mitochondrial-mediated pathway in human uterine carcinosarcoma. Cancer Biol Ther 8: 533-539, 2009.
16. You H, Yoon HE, Yoon JH, Ko H and Kim YC: Synthesis of pheophorbide-a conjugates with anticancer drugs as potential cancer diagnostic and therapeutic agents. Bioorg Med Chem 19: 5383-5391, 2011.

17. Lim DS, Ko SH and Lee WY: Silkworm-pheophorbide alpha mediated photodynamic therapy against B16F10 pigmented melanoma. J Photochem Photobiol B 74: 1-6, 2004.

18. Stiel H, Marlow I and Roeder B: Photophysical properties of the photosensitizer pheophorbide a studied at high photon flux densities. J Photochem Photobiol B 17: 181-186, 1993.

19. Hoi SW, Wong HM, Chan JY, et al: Photodynamic therapy of pheophorbide a inhibits the proliferation of human breast tumour via both caspase-dependent and -independent apoptotic pathways in in vitro and in vivo models. Phytother Res: Nov 10, 2011 (Epub ahead of print). doi: $10.1002 /$ ptr.3607.

20. Chung IS, Son YI, Ko YJ, Baek CH, Cho JK and Jeong HS: Peritumor injections of purified tumstatin delay tumor growth and lymphatic metastasis in an orthotopic oral squamous cell carcinoma model. Oral Oncol 44: 1118-1126, 2008.

21. Pang S, Kang MK, Kung S, et al: Anticancer effect of a lentiviral vector capable of expressing HIV-1 Vpr. Clin Cancer Res 7: 3567-3573, 2001.

22. Herzog M, Moser J, Wagner B and Broecker J: Shielding effects and hypoxia in photodynamic therapy. Int J Oral Maxillofac Surg 23: 406-408, 1994.

23. Uehara M, Ikeda $\mathrm{H}$, Nonaka $\mathrm{M}$, et al: Predictive factor for photodynamic therapy effects on oral squamous cell carcinoma and oral epithelial dysplasia. Arch Oral Biol 56: 1366-1372, 2011.

24. Lu Z, Tao Y,Zhou Z, et al: Mitochondrial reactive oxygen species and nitric oxide-mediated cancer cell apoptosis in 2-butylamino2-demethoxyhypocrellin B photodynamic treatment. Free Radic Biol Med 41: 1590-1605, 2006.

25. Smiley ST, Reers M, Mottola-Hartshorn C, et al: Intracellular heterogeneity in mitochondrial membrane potentials revealed by a J-aggregate-forming lipophilic cation JC-1. Proc Natl Acad Sci USA 88: 3671-3675, 1991.

26. Tomioka Y, Kushibiki T and Awazu K: Evaluation of oxygen consumption of culture medium and in vitro photodynamic effect of talaporfin sodium in lung tumor cells. Photomed Laser Surg 28: 385-390, 2010.

27. Nowis D, Makowski M, Stoklosa T, Legat M, Issat T and Golab J: Direct tumor damage mechanisms of photodynamic therapy. Acta Biochim Pol 52: 339-352, 2005.

28. Rapozzi V, Miculan M and Xodo LE: Evidence that photoactivated pheophorbide a causes in human cancer cells a photodynamic effect involving lipid peroxidation. Cancer Biol Ther 8: 1318-1327, 2009.

29. Garg TK and Chang JY: 15-deoxy-delta 12, 14-Prostaglandin J2 prevents reactive oxygen species generation and mitochondrial membrane depolarization induced by oxidative stress. BMC Pharmacol 4: 6, 2004

30. Tang PM, Zhang DM, Xuan NH, et al: Photodynamic therapy inhibits P-glycoprotein mediated multidrug resistance via JNK activation in human hepatocellular carcinoma using the photosensitizer pheophorbide a. Mol Cancer 8: 56, 2009.

31. Foster TH, Giesselman BR, Hu R, Kenney ME and Mitra S: Intratumor administration of the photosensitizer pc 4 affords photodynamic therapy efficacy and selectivity at short drug-light intervals. Transl Oncol 3: 135-141, 2010.

32. Griffin GM, Zhu T, Solonenko M, et al: Preclinical evaluation of motexafin lutetium-mediated intraperitoneal photodynamic therapy in a canine model. Clin Cancer Res 7: 374-381, 2001.

33. Perry RR, Smith PD, Evans S and Pass HI: Intravenous vs intraperitoneal sensitizer: implications for intraperitoneal photodynamic therapy. Photochem Photobiol 53: 335-340, 1991. 